\title{
NOVEL SEMG PARAMETERS FOR EARLY DIAGNOSTICS OF NEUROLOGICAL DISEASES AND AGING
}

\author{
Alexander Yu. Meigal ${ }^{1}$, Saara M. Rissanen ${ }^{2}$, Mika P. Tarvainen ${ }^{2}$, Verneri Ruonala ${ }^{2}$, \\ Olavi Airaksinen ${ }^{3}$, Markku Kankaanpää ${ }^{4}$, German G. Miroshnichenko ${ }^{1,2}$, Anna P. Kuzmina ${ }^{1}$, \\ Pasi A. Karjalainen ${ }^{2}$ \\ ${ }^{1}$ Institute of High-Tech Biomedicine, Petrozavodsk State University, Petrozavodsk, Russia \\ ${ }^{2}$ Department of Applied Physics, University of Eastern Finland, Kuopio, Finland \\ ${ }^{3}$ Department of Physical Medicine and Rehabilitation, Kuopio University Hospital, Kuopio, \\ Finland \\ ${ }^{4}$ Department of Physical Medicine and Rehabilitation, Tampere University Hospital, \\ Tampere, Finland
}

A.Yu. Meigal

33 Lenin Avenue, Petrozavodsk, Russia 185910

$\underline{\text { meigal@petrsu.ru }}$

\begin{abstract}
The early-stage diagnostics is critical for early treatment of neurodegenerative diseases. Although Parkinson's disease (PD) as one of the most studied neurological diseases has been intensively studied, the pre-clinical markers of PD is yet to be established. Here we report on the non-linear characteristics of surface electromyogram (sEMG) and tremor acceleration as a possible diagnostic tool, and, in prospective, as a predictor for PD. We found that such nonlinear parameters as percent of determinism, sample entropy and correlation dimension allowed discriminating some $85 \%$ of healthy controls from PD patients. We intend to expand this approach to multiply sclerosis, schizophrenia and essential tremor.
\end{abstract}

Key words: Parkinson's disease, electromyography, nonlinear parameters, early diagnostics, ageing. 


\section{Why to search for novel biosignals and early diagnostics in neurology?}

\subsection{Economical and personal burden.}

Neurodegenerative diseases, such as Alzheimer's disease (AD), Parkinson's disease (PD), multiply sclerosis (MS), along with schizophrenia (SZ), essential tremor (ET) and Huntington's disease (HD) imply strong economical burden of several kinds on the patients, their relatives and society. In the USA at present, AD afflicts $13 \%$ of people aged over 65 and $43 \%$ of those 85 and older (in total, an estimated 5.4 million) (Ambrose, 2013). PD is the second most common, after $\mathrm{AD}$, neurodegenerative disease. The incidence of PD in developed countries is estimated at about $1 \%$ in people older than 60 years and $4 \%$ in people over 80 years (de Lau, 2006). SZ affects approximately $1 \%$ of the population but SZ patients occupy $25 \%$ of all hospital beds in the USA (Davies, 1994). The number of people with MS is 2-2.5 million globally, with rates varying from 0.5 per 100,000 in Africa to 200 per 100,000 in certain populations of Northern Europe (Adelman, 2013).

The personal burden means dramatic decrease of the quality of the patient's life due to motor (tremor, rigidity, akynesia, atonia, postural instability, fatigue, and muscle weakness) and non-motor symptoms (impaired autonomic functions, depression, sleep, emotional and cognitive disorders). These motor and non-motor symptoms may decrease person's ability to work and thus may imply early retirement, restrictions on profession choice and, hence, the salary ( Keränen, 2003). Cost burden of neurological patients also includes medication, care costs, insurance etc. For example, annual economical burden on one PD patient in the developed countries exceeds 600025000 USD (Noyes, 2006; Tolosa, 2006), and on one MS patient 8000-50000 USD (Adelman, 2013). The overall U.S. 2002 cost of schizophrenia was estimated to be 62.7 billion USD (van den Heuvel, 2010).

These diseases, in a sense, are "non-lethal" pathologies because they do not cause immediate fatal outcome. They can last, like PD under medication, for decades. The average life expectance of men with SZ is only slightly lower than would be expected for the population as a whole (Davies, 1994).

\subsection{Early diagnosis.}

In some neurological patients, diagnosis is highly uncertain, especially in the early stages. For example, only $70 \%$ of patients are correctly diagnosed with PD (Tolosa, 2006). PD, ET, MS, $\mathrm{AD}$ and HD share many motor symptoms among each other. There is a large body of papers on comparative studies of PD and ET (Becker, 2002). However, a diagnostic tool to differentiate these two pathologies is yet to be elaborated.

Neurodegenerative diseases are characterized by a lengthy prodromal due to their chronic course, which is known as "preclinical" phase (Berendse, 2006). For example, PD becomes clinically recognizable only when $60 \%$ of dopaminergic neurons in the compact part of substantia nigra are lost. This phase is believed to start long before emergence of clinical PD and then may slowly progress over 4.5 years (Postuma, 2013). The prodromal period presents unique opportunity in disease prevention or delay in PD onset. Some $10 \%$ of subjects over 60 years are in the "prediagnostic" phase of PD according to neuropathological reports (Stern, 2004). Therefore, predating the diagnosis of PD and identifying subjects at risk is an important goal for research aimed to postpone the onset of PD by neuroprotective therapy (Siderowf, 2006). Thus, the ultimate goal of the early diagnosis of PD would be switch from medical treatment to the disease management. 
A predictive study would help to identify the subjects at-risk of future disease, to start earlier treatment, and to develop effective neuroprotective treatment strategies (Siderowf, 2006). Here, we report on current approaches in early diagnostics and differential diagnosis of PD with special stress on the nonlinear parameters of interference surface electromyography (sEMG) signal.

\section{Current approaches to early diagnosis of neurological disease.}

The preclinical (early clinical) predictors become a hallmark of the current neurology, which would present a window of therapeutic opportunity for many neurological diseases. PD is one of the best studied diseases in respect with early diagnosis. Visual, smell, sleep, vocal, handwriting, autonomic, motor and mental dysfunction due to dopaminergic deficit affect $60-80 \%$ of PD patients (Tolosa, 2006) and they are usually synchronized. These are used as prodromal markers for PD. However, definitive PD-sensitive diagnostic tool is lacking. So, the decisive diagnosis of PD is still post-mortem. All in all, current methods help to diagnose only $70-80 \%$ of the PD cases (Becker, 2002; Berendse, 2006; Müller, 2001; Tissingh, 2001), which is not satisfactory. It corresponds with misdiagnosis of PD estimated as 20-30\% (Jankovic, 2008). Novel biomarkers for PD must be presented. Over the last years, single photon emission computed tomography (SPECT), positron emission tomography (PET), fMRI and transcranial sonography are widely used to assess dopaminergic function in PD patients, their relatives and healthy controls (Becker, 2002; Müller, 2001). However, their diagnostic yield is still far from obvious (Morrish, 1997). For example, up to $15 \%$ of subjects with normal imaging findings, have clinically evident PD, and vise versa, decreased dopamine content in basal ganglia seen on MRI is often associated with neurodegenerative diseases other than PD (Bajaj, 2013). Also, methods based on nuclear medicine and ultrasound are not appropriate for population-based studies due to their high costs, and insufficient availability (Becker, 2002; Müller, 2001). The concept of a preclinical phase of disease is not foreign also for AD (Nitrini, 2010; Sperling, 2011) and MS (Andersen, 2010).

\section{EMG as a potential early marker for PD.}

To our mind, the method for early diagnostics of neurological disease must be sensitive, cost-efficient, non-invasive, high throughput and non-hazardous. We believe that surface electromyography (sEMG) is such a method. EMG is a kind of "mirror" from skeletal muscles for central nervous system (CNS) because it reflects the activity of the spinal motoneurons due to motor units (MUs). No consensus exists about applicability of spectral-based analysis of sEMG to PD diagnosis because conventional linear parameters are still lacking to provide definitive difference between the PD and healthy controls.

The morphology-based analysis has shown promising results in discriminating PD and healthy controls. The method is based on the histogram and crossing rate analysis of sEMG signals (Rissanen, 2007). sEMG kurtosis, a parameter based on higher order statistics, is reportedly increased in PD patients, that might reflect increased number of spikes due to increased synchronicity of MU firing (Meigal, 2009). Also, previous works have reported that sEMG waveform can better be modeled as an output of a nonlinear dynamic system, rather than a stochastic output of a white-noise driven linear system (Rissanen, 2007). Nonlinearity is a hallmark of complex dynamic systems (Elbert, 1994; Stam, 2007). As a nonlinear signal, sEMG displays chaotic behavior, i.e. its time series 1) evolves over the time, 2) depends on the initial state, and 3) is fractal in the terms of dimensionality (Nieminen, 1996). Thus, as a nonlinear signal, sEMG can be characterized by the state of deterministic chaos (Webber, 1995). Therefore, sEMG might give clues to describe dynamics of the neuronal circuits in the terms of regularity, predictability, and complexity (Goldberger, 2002). Indeed, it has been recently found that such nonlinear parameters as 
approximate entropy, percent of determinism based on recurrence quantification analysis (RQA), and dimensionality based on fractal analysis are highly sensitive for hidden rhythms on sEMG in subjects under fatigue and condition of increased MU synchronization (Del Santo, 2007).

sEMG in the PD patients is known to be rich in regular clusters (grouping) at the characteristic tremor frequencies (4-6 Hz) due to increased synchronization of MU (Vaillancourt, 2000). Acceleration signal has also been studied in PD patients using both linear and nonlinear parameters (Sturman, 2005; Vaillancourt, 2000; Vaillancourt, 2003). These findings led us ask whether either readily visible or "hidden" rhythms in sEMG contribute to its nonlinear features and thus yield difference between the PD patients and healthy controls? If this hypothesis holds, the PD patients might present a more regular time-dependent structure of sEMG and acceleration time series, while healthy subjects - a less regular and more complex signal. Also, PD patients with less expressed sEMG clustering might present less regular signal. To test this hypothesis, we compared a variety of novel nonlinear parameters with the classic linear parameters between PD patients with various UPDRS scores, healthy young and older controls.

We found that nonlinear parameters of sEMG signal in the PD group significantly differed from the ones in the healthy control groups (Meigal, 2009). In particular, percent of determinism (\%DET) values of sEMG were significantly higher in the PD group, while sample entropy (SampEn) and correlation dimension (CD) were lower in comparison to old and young controls. Instead, such traditional parameters as RMS and MDF did not differ between groups. Decreased CD of sEMG in the PD group may indicate increased self-similarity of the myoelectrical signal over time (Siderowf, 2006) and hence, lowered complexity of the underlying neural network. Decreased SampEn of sEMG signal in the PD group may reflect higher regularity of sEMG. High \%DET reflects abundant wave features in sEMG, either readily visible by eye or quasi-waves during, e.g. increased motor unit synchronization (Rissanen, 2007; Rissanen, 2009). Clustering of MU action potentials was the characteristic of raw sEMG of many our PD patients (Meigal, 2009). Interestingly, \%DET also was increased in some patients with visually stationary sEMG. We have also found that such sEMG parameters, as \%REC, and \%DET were correlated with Unified Parkinson's Disease Rating Scale (UPDRS) score. Most of novel sEMG characteristics correlated also with finger tapping scores (Meigal, 2009). Correlation values were the most significant in the state without additional loading, and they decreased when loading increased. This probably indicates emergence of "regular normal" postural muscle tonus, which erased the difference between the groups. In a sense, under loading sEMG of PD patients became more "normal". Thus, sEMG signal in PD is less complex, more predictable and regular. It means that rhythmic activity takes place in the spinal cord, resulting in more or less obvious clustering of sEMG. From the physiological point of view, these data reflect increased synchronization of MU activity or increased clustering coefficient of signal generator (the spinal neuron circuitry) (Stam, 2007). It could also well be that the spinal cord rather relays this periodicity from the upper levels of CNS, than generates it itself.

\section{4. sEMG parameters in SZ patients.}

Investigations of nonlinearity of biosignals in SZ are usually focused on brain and EEG. For example, application of graph theory revealed that brain network is substantially disintegrated in SZ (Wu, 2005). Muscles may reflect aberrant descending motor flow thus forming a hypothetical "muscle channel" for sEMG diagnostics of SZ. Dopamine hypothesis of SZ argues that its symptoms can be explained by elevated level of dopamine in the brain (Carlsson, 1963). As such, one can suggest that SEMG parameters must be opposite to those found in PD, at which dopamine production is decreased (Hornykiewicz, 1975). Namely, we expect that sEMG in SZ patients might have been characterized by high entropy and correlation dimension, and low percent of determinism. Further measurements would support or refute that assumption. 


\section{Non-linear parameters of sEMG under ageing.}

Because of overwhelming prevalence of movement disorders among elderly individuals, it is important to distinguish healthy aging from neurological pathologies (Sturman, 2005). Studies, in which the linear parameters of sEMG were used, showed that EMG signal changes over lifetime (Gerasimova, 2004). There is also a strong conviction that biosignal in human organism become simpler over the lifetime (Goldberger, 2002) due to either loss of CNS oscillators over lifetime or losing capability to balance these oscillators (Vaillancourt, 2003). The outcome of such condition would be dominance of one (few) oscillators which are able to express their own rhythm on sEMG. However, we did not observe reduced complexity of sEMG signal in older controls (Meigal, 2009; Meigal, 2012). Moreover, SampEn and CD were even slightly elevated in aged group, though nonsignificantly (Figure). It means that in old controls sEMG signal was even more complex in comparison to PD patients and young controls. Thus, nonlinear parameters might help to distinguish between pathologically affected and "normal" sEMG samples. By now, our methods allow to discriminate almost $87 \%$ of PD patients from healthy controls (Rissanen, 2009). It could well be that trajectory of complexity over lifetime bifurcates at definite age forming two new trajectories one for "pathology" and another - for "normal" ageing. Such trajectory may have diagnostic value to predict pathological changes of the motor system.

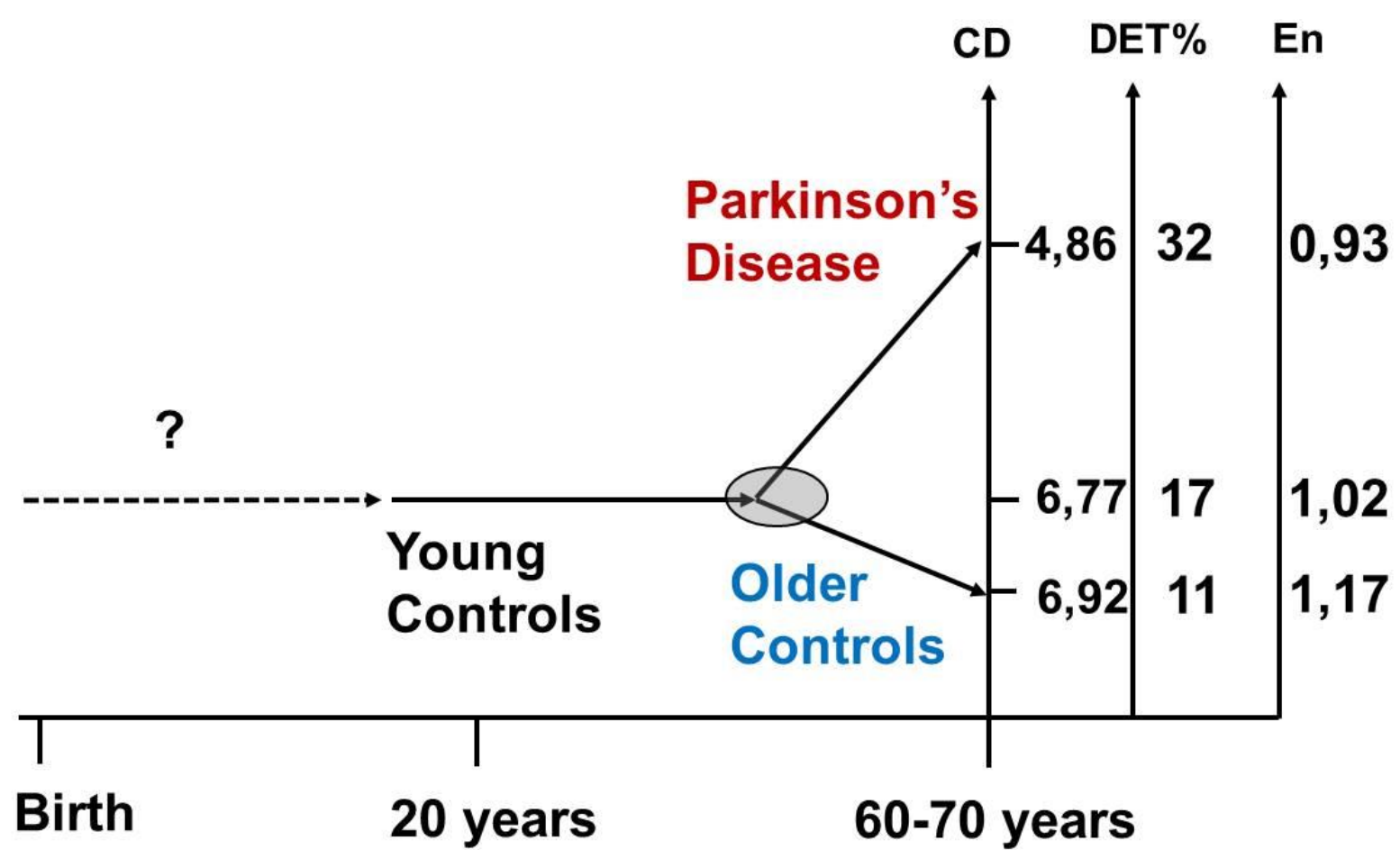

Figure. Trajectories of the nonlinear sEMG parameters over lifetime. Bifurcation of trajectories is denoted as a light grey circle. Difference is statistically significant between PD patients and young controls, but it was not significant between young and older controls.

CD - correlation dimension, DET\% - percent of determinism, En - sample entropy.

\section{Conclusion}

The novel nonlinear sEMG parameters have potential in the preclinical diagnosis of PD due to their relatively high discrimination power, cost efficiency and high throughput. Additionally, nonlinear parameters of sEMG may help to distinguish between healthy ageing and pathology 
during elderly age. Finally, we believe that sEMG could be helpful in early detection of other neurodegenerative diseases, such as $\mathrm{AD}, \mathrm{MS}$, and also SZ.

\section{Acknowledgments}

This study was supported by the Academy of Finland under Project 252748 and Program of strategic development of Petrozavodsk State University (2012-2016, project № 01201372071).

Conflict of interests: the research was conducted in the absence of any commercial or financial relationships that could be construed as a potential conflict of interest.

\section{References}

1. Adelman G, Rane SG, Villa KF. The cost burden of multiple sclerosis in the United States: a systematic review of the literature. J Med Econ 2013, 16:639-647.

2. Ambrose CT. Alzheimer's Disease: The Great Morbidity of the 21 st Century. American Scientist 2013, 101:194-201.

3. Andersen O. Predicting a window of therapeutic opportunity in multiple sclerosis. Brain 2010, 133:1863-1868.

4. Bajaj N, Hauser RA, Grachev ID. Clinical utility of dopamine transporter single photon emission CT (DaT-SPECT) with (123I) ioflupane in diagnosis of parkinsonian syndromes. $J$ Neurol Neurosurg Psychiatry 2013, 84:1288-1295.

5. Becker G, Müller A, Braune S, Büttner T, Benecke R, Greulich W, Klein W, Mark G, Rieke J, Thümler R. Early diagnosis of Parkinson's disease. J Neurol 2002, 249(Suppl3):III/40-48.

6. Berendse HW, Ponsen MM. Detection of preclinical Parkinson's disease along the olfactory tract. J Neural Transm 2006, 70:321-325.

7. Büttner T, Kuhn W, Patzold T, Przuntek H. L-Dopa improves colour vision in Parkinson's disease. J Neural Transm Park Dis Dement Sect 1994, 7:13-19.

8. Carlsson A, Lindquist M. Effect of chlorpromazine or haloperidol on the formation of 3methoxytyramine and normetanephrine in mouse brain. Acta Pharmacol Toxicol (Copenh) 1963, 20:140-144.

9. Davies LM, Drummond MF. Economics and schizophrenia: the real cost. $J$ Psychiatry Suppl. 1994, 25:18-21.

10. de Lau LM, Breteler MM. Epidemiology of Parkinson's disease. Lancet Neurol 2006, $5: 525-535$.

11. Del Santo F, Gelli F, Mazzocchio R, Rossi A. Recurrence quantification analysis of surface EMG detects changes in motor unit synchronization induced by recurrent inhibition. Exp Brain Res 2007, 178:308-315.

12. Elbert T, Ray WJ, Kowalik ZJ, Skinner JE, Graf KE, Birbaumer N. Chaos and physiology: deterministic chaos in excitable cell assemblies. Physiol Rev 1994, 74:1-47.

13. Gerasimova LI, Varlamova TV, Antonen EG, Antropova ES, Meigal AIu. Agerelated changes in turn-amplitude characteristics of the electromyogram recorded during graded isometric contraction. Fiziol Cheloveka (Russian) 2004, 30:119-25.

14. Goldberger AL, Amaral LA, Hausdorff JM, Ivanov PCh, Peng CK, Stanley HE. Fractal dynamics in physiology: alterations with disease and aging. Proc Natl Acad Sci 2002, 99.Suppl 1:2466-2472.

15. Hornykiewicz O. Brain monoamines and parkinsonism. Natl Inst Drug Abuse Res Monogr Ser 1975, 3:13-21.

16. Jankovic J. Parkinson's disease: clinical features and diagnosis. J Neurol Neurosurg Psychiatry 2008, 79:368-376.

17. Keränen T, Kaakkola S, Sotaniemi K, Laulumaa V, Haapaniemi T, Jolma T, Kola H, Ylikoski A, Satomaa O, Kovanen J, Taimela E, Haapaniemi H, Turunen H, Takala A. Economic 
burden and quality of life impairment increase with severity of PD. Parkinsonism Relat Disord 2003, 9:163-168.

18. Meigal AI, Rissanen S, Tarvainen MP, Karjalainen PA, Iudina-Vassel IA, Airaksinen O, Kankaanpää M. Novel parameters of surface EMG in patients with Parkinson's disease and healthy young and old controls. J Electromyogr Kinesiol 2009, 19: e206-213.

19. Meigal AY, Rissanen SM, Tarvainen MP, Georgiadis SD, Karjalainen PA, Airaksinen O, Kankaanpää M. Linear and nonlinear tremor acceleration characteristics in patients with Parkinson's disease. Physiol Meas 2012, 33:395-412.

20. Morrish PK. Parkinson's disease is not a long-latency illness. Mov Disord 1997, 12:849-854.

21. Müller V, Lutzenberger W, Pulvermüller F, Mohr B, Birbaumer N. Investigation of brain dynamics in Parkinson's disease by methods derived from nonlinear dynamics. Exp Brain Res 2001, 137:103-110.

22. Nieminen H, Takala EP. Evidence of deterministic chaos in the myoelectric signal. Electromyogr Clin Neurophysiol 1996, 36:49-58.

23. Nitrini R. Preclinical diagnosis of Alzheimer's disease: Prevention or prediction? Dement Neuropsychol 2010, 4:259-261

24. Noyes K, Liu H, Li Yu, Holloway R, Dick AW. Economic burden associated with Parkinson's disease on elderly Medicare beneficiaries. Mov Disord 2006, 21:362-372.

25. Postuma RB, Gagnon JF, Montplaisir JY. REM sleep behavior disorder and prodromal neurodegeneration - Where are we headed? Tremor Other Hyperkinet Mov (N Y) 2013, 3: doi: pii:tre-03-134-2929-1.

26. Rissanen S, Kankaanpää M, Tarvainen MP, Nuutinen J, Tarkka IM, Airaksinen O, Karjalainen PA. Analysis of surface EMG signal morphology in Parkinson's disease. Physiol Meas 2007, 8:1507-1521.

27. Rissanen SM, Kankaanpää M, Tarvainen MP, Meigal AY, Nuutinen J, Tarkka IM, Airaksinen O, Karjalainen PA. Analysis of dynamic voluntary muscle contractions in Parkinson's disease. IEEE Trans Biomed Eng 2009, 56:2280-2288.

28. Siderowf A, Stern MB. Preclinical diagnosis of Parkinson's disease: are we there yet? Curr Neurol Neurosci Rep 2006, 6:295-301.

29. Sperling RA, Aisen PS, Beckett LA. Toward defining the preclinical stages of Alzheimer's disease: Recommendations from the National Institute on Aging and the Alzheimer's Association workgroup Alzhemer's and Dementia 2011, 7(3):1-13.

30. Stam CJ, Reijneveld JC. Graph theoretical analysis of complex networks in the brain. Nonlinear Biomed Phys 2007, 1:3.

31. Stern MB. The preclinical detection of Parkinson's disease: ready for prime time? Ann Neurol 2004, 56:169-171.

32. Sturman MM, Vaillancourt DE, Corcos DM. Effects of aging on the regularity of physiological tremor. J Neurophysiol 2005, 93:3064-3074.

33. Tissingh G, Berendse HW, Bergmans P, DeWaard R, Drukarch B, Stoof JC, Wolters EC. Loss of olfaction in de novo and treated Parkinson's disease: possible implications for early diagnosis. Mov Disord 2001, 16:41-46.

34. Tolosa E, Wenning G, Poewe W. The diagnosis of Parkinson's disease. Lancet Neurol 2006, 5:75-86.

35. Vaillancourt DE, Larsson L, Newell KM. Effects of aging on force variability, single motor unit discharge patterns, and the structure of 10, 20 and $40 \mathrm{~Hz}$ EMG activity. Neurobiol Aging $2003,24: 25-35$.

36. Vaillancourt DE, Newell KM. The dynamics of resting and postural tremor in Parkinson's disease. Clin Neurophysiol 2000, 111:2046-2056.

37. Webber CL Jr, Schmidt MA, Walsh JM. Influence of isometric loading on biceps EMG dynamics as assessed by linear and nonlinear tools. J Appl Physiol 1995, 78:814-822. 
38. van den Heuvel MP, Mandl RC, Stam CJ, Kahn RS, Hulshoff Pol HE. Aberrant frontal and temporal complex network structure in schizophrenia: a graph theoretical analysis. $J$ Neurosci 2010, 30:15915-15926.

39. Wu EQ, Birnbaum HG, Shi L, Ball DE, Kessler RC, Moulis M, Aggarwal J. The economic burden of schizophrenia in the United States in 2002. J Clin Psychiatry 2005, 66:11221129. 